\title{
Flexible CFD meshing strategy for prediction of ship resistance and propulsion performance
}

\author{
Jeong Hwa Seo ${ }^{1}$, Dong Myung Seol ${ }^{1}$, Ju Hyun Lee ${ }^{1}$, Shin Hyung Rhee ${ }^{1,2}$ \\ ${ }^{I}$ Department of Naval Architecture and Ocean Engineering, Seoul National University, Seoul, Korea \\ ${ }^{2}$ Research Institute of Marine Systems Engineering, Seoul National University, Seoul, Korea
}

\begin{abstract}
In the present study, we conducted resistance test, propeller open water test and self-propulsion test for a ship's resistance and propulsion performance, using computational fluid dynamics techniques, where a Reynolds-averaged NavierStokes equations solver was employed. For convenience of mesh generation, unstructured meshes were used in the bow and stern region of a ship, where the hull shape is formed of delicate curved surfaces. On the other hand, structured meshes were generated for the middle part of the hull and the rest of the domain, i.e., the region of relatively simple geometry. To facilitate the rotating propeller for propeller open water test and self-propulsion test, a sliding mesh technique was adopted. Freesurface effects were included by employing the volume of fluid method for multi-phase flows. The computational results were validated by comparing with the existing experimental data.
\end{abstract}

KEY WORDS: Flexible meshing; Resistance performance; Self-propulsion performance; Propeller open water performance; Computational fluid dynamics.

\section{INTRODUCTION}

Thanks to the great advancement of computer performance recently, computational analysis of ship resistance and propulsion using computational fluid dynamics (CFD) is being widely adopted, and the results are being applied to actual design of ships (Yang et al., 2009; Jasak, 2009; Kim et al., 2010; Seo et al., 2010). In those applications, however, the difficulty in mesh generation is the most serious obstacle for non-expert users for utilizing CFD with maximum efficiency. In case of mesh generation around a ship hull, the bow and stern regions require special care and experience, because of the delicate and rapidly changing surfaces. This is true even for a bare hull and the complexity gets out of control, if propellers, rudders, and appendages are included. Therefore, a hybrid meshing approach using unstructured meshing near the complex geometry and structured meshing in the remaining simple geometry domain was suggested(Lee et al., 2009).

In the present study, we extended the application further and executed resistance and self-propulsion tests using hybrid meshing, which employs unstructured meshing in the bow, stern, and propeller region and structured meshing in the remaining region. For propeller open water and selfpropulsion tests, a sliding mesh technique, where the

Corresponding author: Shin Hyung Rhee

E-mail:shr@snu.ac.kr propeller is located in a disk volume and the volume itself rotates with non-conformal interfaces placed between the rotational and stationary sub-domains, was adopted.

The present computational results were investigated through comparison with the experimental datafrom Maritime and Ocean Engineering Research Instituted (MOERI) and other computational results.

\section{COMPUTATIONAL MODEL AND CONDITION}

KRISO container ship (KCS), a container ship designed by MOERI, was selected as the object ship, and KP505, the propeller designed for KCS, was selected as the object propeller. The principal particulars are described in Tables 1 and 2.

Table 1 Principal particulars of KCS.

\begin{tabular}{|l|l|}
\hline Length between perpendiculars & $7.2786 \mathrm{~m}$ \\
\hline Beam & $1.019 \mathrm{~m}$ \\
\hline Draft & $0.3418 \mathrm{~m}$ \\
\hline Wetted surface area & $9.4379 \mathrm{~m}^{2}$ \\
\hline Speed & $2.196 \mathrm{~m} / \mathrm{s}$ \\
\hline Reynolds number & $1.4 \times 10^{7}$ \\
\hline
\end{tabular}


Table 2 Principal particulars of KP505.

\begin{tabular}{|l|l|}
\hline Diameter & $250.0 \mathrm{~mm}$ \\
\hline Ae/A0 & 0.800 \\
\hline Hub ratio & 0.180 \\
\hline No. of blades & 5 \\
\hline Blade section & NACA66 \\
\hline
\end{tabular}

\section{COMPUTATIONAL METHOD}

\section{Mathematical modeling and Numerical Method}

The governing equations are written for the mass and momentum conservation, such that

$$
\begin{aligned}
& \frac{\partial \rho}{\partial t}+\nabla \cdot(\rho \bar{v})=0 \\
& \frac{\partial}{\partial t}(\rho \bar{v})+\nabla \cdot(\rho \overline{v v})=-\nabla \rho+\nabla \cdot \overline{\bar{\tau}}+\rho g
\end{aligned}
$$

where $\bar{v}$ is the velocity vector in the Cartesian coordinate system, $p$ the static pressure, and $\overline{\bar{\tau}}$ the stress tensor given by

$$
\overline{\bar{\tau}} \equiv \mu\left|\left(\nabla \bar{v}+\nabla \bar{v}^{T}\right)-\frac{2}{3} \nabla \cdot \bar{v} I\right|
$$

Where $\mu$ is the molecular viscosity, $I$ the unit tensor, and the second term on the right hand side the effect of volume dilation. Once the Reynolds averaging approach for turbulence modeling is applied, the Navier-Stokes equations can be written in Cartesian tensor form as

$$
\begin{aligned}
& \frac{\partial}{\partial t}\left(\rho u_{i}\right)+\frac{\partial}{\partial x_{j}}\left(\rho u_{i} u_{j}\right) \\
& =-\frac{\partial \rho}{\partial x_{i}}+\frac{\partial}{\partial x_{j}}\left[\mu\left(\frac{\partial u_{i}}{\partial x_{j}}+\frac{\partial u_{j}}{\partial x_{i}}-\frac{2}{3} \delta_{i j} \frac{\partial u_{i}}{\partial x_{i}}\right)\right]+\frac{\partial}{\partial x_{j}}\left(-\rho \bar{u}_{i}^{\prime} \bar{u}_{j}^{\prime}\right)
\end{aligned}
$$

where $\delta_{i j}$ is the Kronecker delta, and $-\rho \overline{u_{\imath}^{\prime} u_{\jmath}^{\prime}}$ the Reynolds stresses. These Reynolds stresses must be modeled to close equation (4), i.e., for turbulence closure.

The volume of fluid (VOF) method is employed to handle the free-surface wave flow around the ship. Based on the local volume fraction of the $q$-th fluid, $\alpha_{q}$, the appropriate variables and properties are assigned to each cell within the domain. The tracking of the interfaces between the phases is accomplished by the solution of a continuity equation for the volume fraction of phases. For the $q$-th phase, this equation has the following form

$$
\frac{\partial \alpha_{q}}{\partial t}+\bar{v} \cdot \nabla \alpha_{q}(\rho \bar{v})=0
$$

Note that the volume fraction equation is not solved for the primary phase, but based on the following constraint

$\sum_{q=1}^{n} \alpha_{q}=1$

A single momentum equation is solved throughout the domain, and the resulting velocity field is shared among the phases. The momentum equation depends on the volume fractions of all phases through the fluid properties, which are determined by the presence of the component phases in each cell, e.g.,

$\rho=\sum_{q=1}^{n} \alpha_{q} \rho_{q}$

In the case of turbulence quantities, a single set of transport equations is solved, and the turbulence variables are shared by the phases throughout the field.

The computations were carried out using FLUENT v6.3, general purpose CFD software. The governing equations were discretized using implicit unsteady method. The second order accurate upwind scheme was selected for the convection terms, while the second order accurate central scheme was for the diffusion terms. The high resolution interface capturing scheme (HRIC) was used for the discretization of the volume fraction equation near the interface. For the velocity and pressure coupling, the SIMPLEC algorithm was used and the k- $\varepsilon$ turbulence model with a wall function was used for turbulence closure. To confirm the convergence, averaged residuals scaled by the initial imbalance of equations were checked. The criterion for the residual was $10^{-6}$. In addition, the constancy of the total force on the ship or propeller was chosen as another standard for confirming the convergence.

\section{Computational Mesh and Boundary Conditionsfor Resistance Test}

The computational domain is shown in Fig. 1 with its dimensions. In order to accommodate the free surface motion, there is vertical room of $0.047 \mathrm{~L}$ between the top boundary and initial water surface.

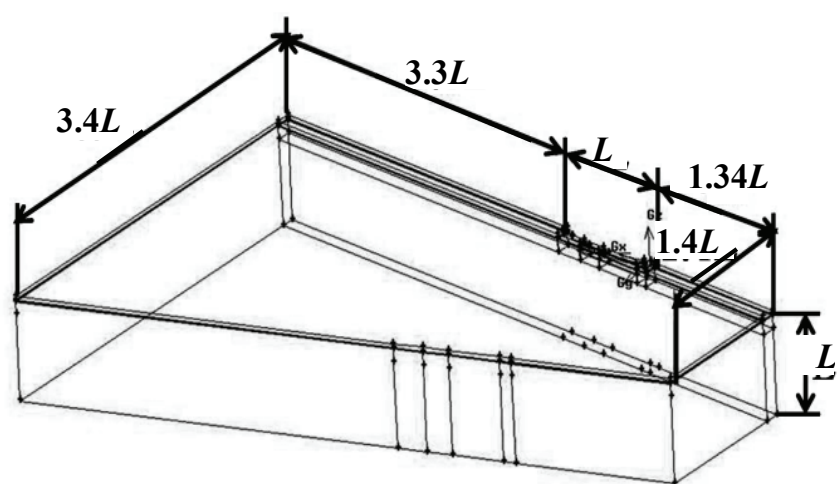

Fig. 1 Computational domain for resistance test. 
The no-slip condition was applied on the hull surface. On the upstream and side boundaries, the total pressure including hydrostatic one was applied, while the static pressure was extrapolated through the downstream boundary. The top and bottom boundaries were considered as free slip wall.

Fig. 2 shows the mesh on the hull surface. Unstructured meshing was used in the bow and stern region, while structured meshing was applied in the middle part of the hull. Between the unstructured and structured mesh sub-domains, non-conformal interfaces were placed. The total number of cells used for resistance test was about 1.56 million, of which 970,000 cells were tetrahedrons and 590,000 cells were hexahedrons.

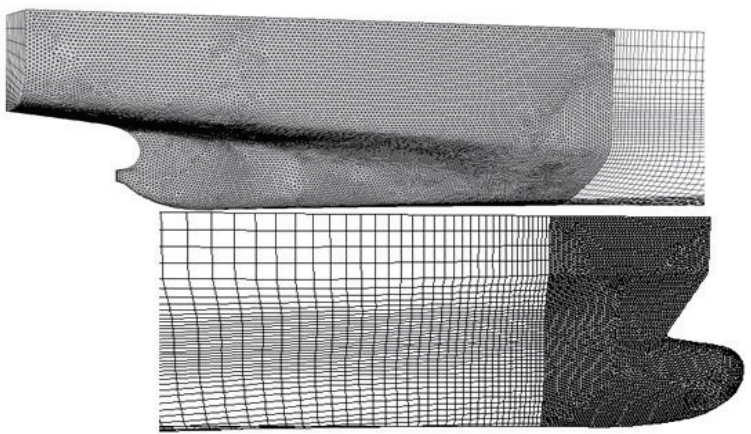

Fig. 2 Hull surface mesh.

\section{Computational Mesh and Boundary Conditions for Propeller Open Water Test}

The computational domain around the propeller and its dimensions are shown in Fig. 3. On the propeller and its hub, no-slip condition was applied. On the inlet boundary, velocity components of uniform stream with the given inflow speed were imposed, while the static pressure was set to zero on the exit boundary. Free slip condition was imposed on the outer cylindrical boundary.

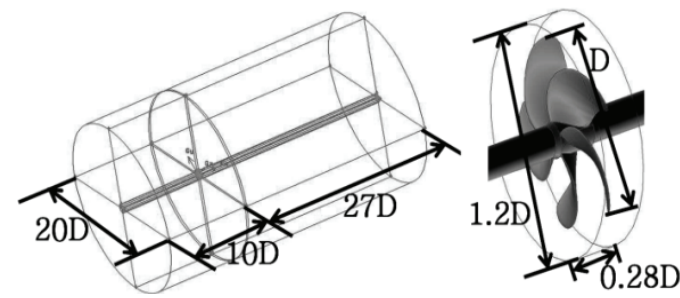

Fig. 3 Computational domain for propeller open water test.

Fig. 4 shows the typical mesh on the cross section across the propeller. Immediately around the propeller, unstructured meshing was used, while the outer part is generated by structured meshing. The interface between the structured and unstructured mesh sub-domains is treated as non-conformal interface. To maintain the first cell height off the solid surface between 30 and 120 in terms of $y^{+}$, four prism layers are applied on the propeller surface, with 138,012prismatic cells. The total number of cells used forpropeller open water test was about 1.43 million, including 756,000 hexahedron cells and 538,530 tetrahedron cells.

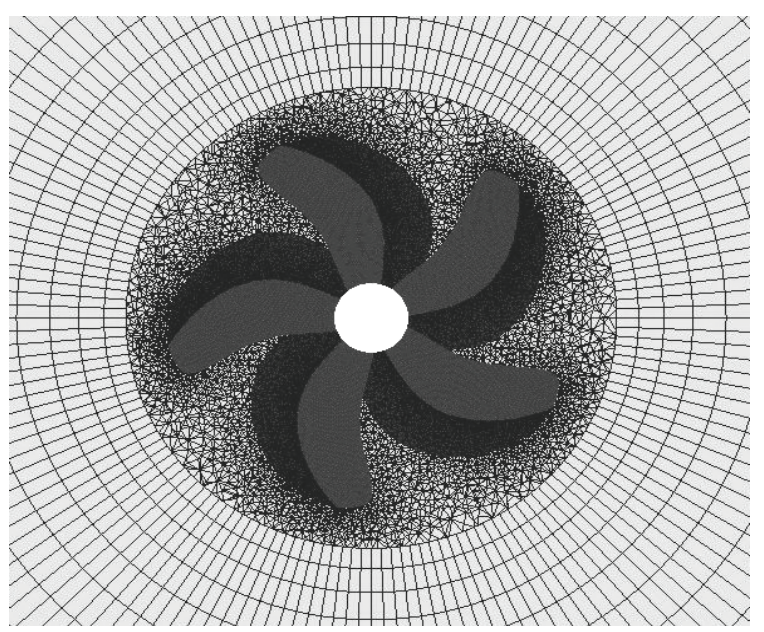

Fig. 4 Propeller mesh.

\section{Computational Mesh and Boundary Conditions for Self- Propulsion Test}

For self-propulsion test, the full domain was used, while the resistance test used the half domain. Fig. 5 shows the domain for self-propulsion test. Vertical room of $0.047 \mathrm{~L}$ for free surface motion exists between the initial water surface and top boundary, as for resistance test. The number of cells for self-propulsion test was about 3.29 million tetrahedrons in the bow, stern and propeller regions, and 1.18million hexahedrons in the remaining part. The total number of the cells for the computation was 4.47 million.

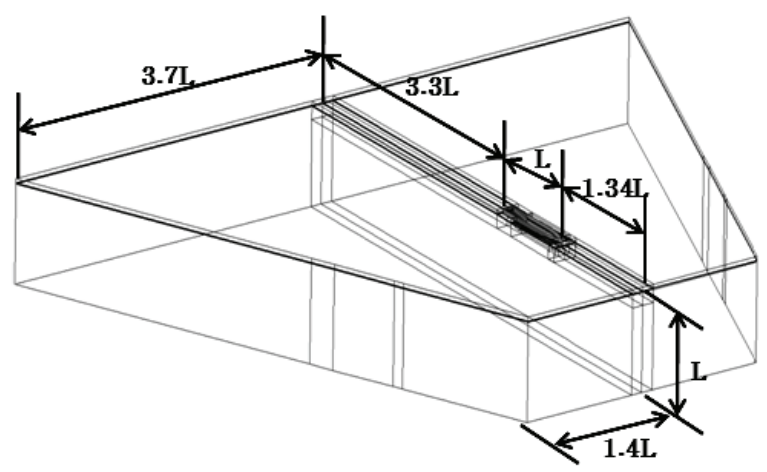

Fig. 5 Computational domain for self propulsion test.

On the hull surface, no-slip condition applied, while the free slip condition is applied on the top and bottom boundaries. On the upstream and side boundaries, total pressure including hydrostatic one was applied. Through the downstream boundary, the static pressure was extrapolated, as for resistance test. To apply non-conformal interface between the propeller region and hull region, the sliding mesh technique was adopted around the propeller as shown in Fig. 6. 


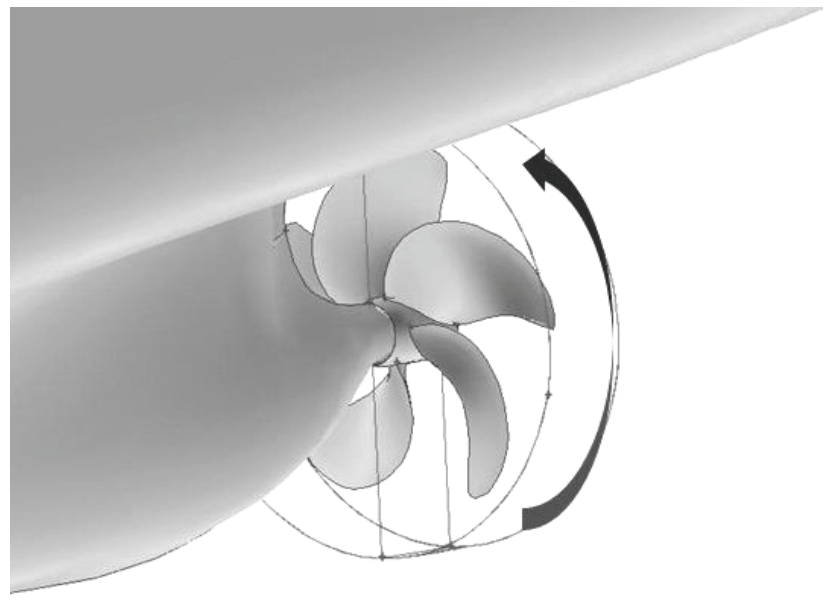

Fig. 6 Configuration of sliding mesh.

\section{RESULTS AND DISCUSSION}

\section{Resistance test}

The resistance test results are summarized in Table 3, showing comparison of the resistance coefficients with the experimental data from MOERI and another set of computational results by WAVIS, a CFD code developed at MOERI (Kim et al., 2005). The total resistance coefficient $\left(C_{T}\right)$ and residuary resistance coefficient $\left(C_{R}\right)$ were compared. Note that the frictional resistance coefficient $\left(C_{F}\right)$ was derived from the ITTC 1957 formula. The overall agreement is very good with close comparison with both experimental and other computational results.

Table 3 Comparison of the Resistance Coefficients.

\begin{tabular}{|l|l|l|l|}
\hline & $\begin{array}{c}\text { MOERI } \\
\text { (Experiment) }\end{array}$ & Present & WAVIS \\
\hline$C_{R} \times 10^{4}$ & 7.316 & $\begin{array}{l}7.197 \\
(-1.63 \%)\end{array}$ & $\begin{array}{l}7.676 \\
(4.92 \%)\end{array}$ \\
\hline \multirow{2}{*}{$C_{T} \times 10^{3}$} & 3.560 & $\begin{array}{l}3.548 \\
(-0.33 \%)\end{array}$ & $\begin{array}{l}3.596 \\
(1.01 \%)\end{array}$ \\
\hline
\end{tabular}

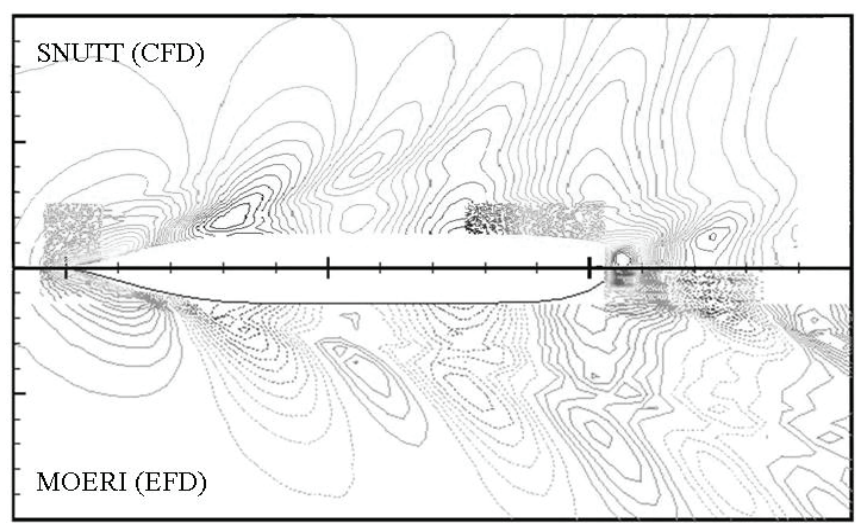

Fig. 7 Comparison of Wave Patterns.
Next, the free-surface wave solutions were compared. Fig. 7 shows the comparison of free-surface wave pattern with the experimental data from MOERI. Although there are irregular contours lines caused by graphical reconstruction of interfaces in tetrahedral cells, the comparison looks quite good, especially near the hull. However, as for other CFD results, the diverging wave away from the hull are underpredicted due to numerical diffusion. Figs. 8 and 9 present the comparison of wave profiles along the hull surface/centerplane and wave cut at $y / L_{p p}=0.1509$. For this comparison, the computational results from National Maritime Research Institute (NMRI) of Japan were used. It is confirmed that, even across the non-conformal interfaces, the free-surface wave solution shows smooth transition and good agreement with the experimental data. As seen in the wave pattern comparison, numerical diffusion causes under-prediction of free-surface wave solution in both the NMRI's and present results.

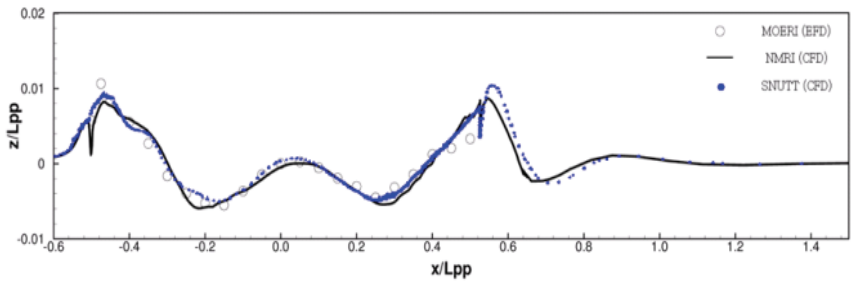

Fig. 8 Comparison of Wave profile.

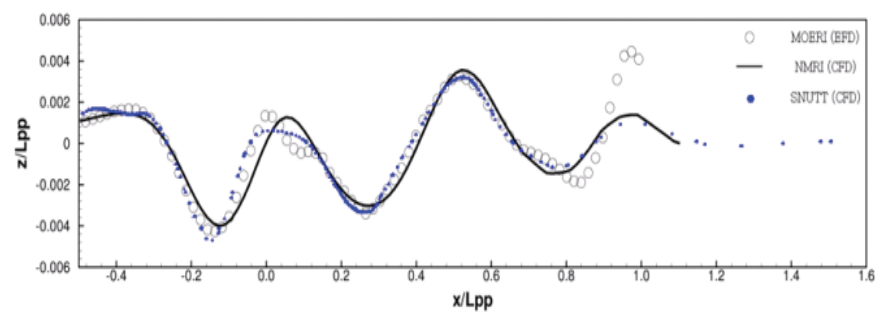

Fig. 9 Comparison of Wave cut at $y / L_{p p}=0.1509$.

The wake distribution at $x / L_{p p}=0.4911$ (from midship) was compared with the experimental data, as shown in Fig. 10.The overall trend was reproduced well, but because of the tetrahedral cells in the region, the strong longitudinal vortices were slightly under-predicted.

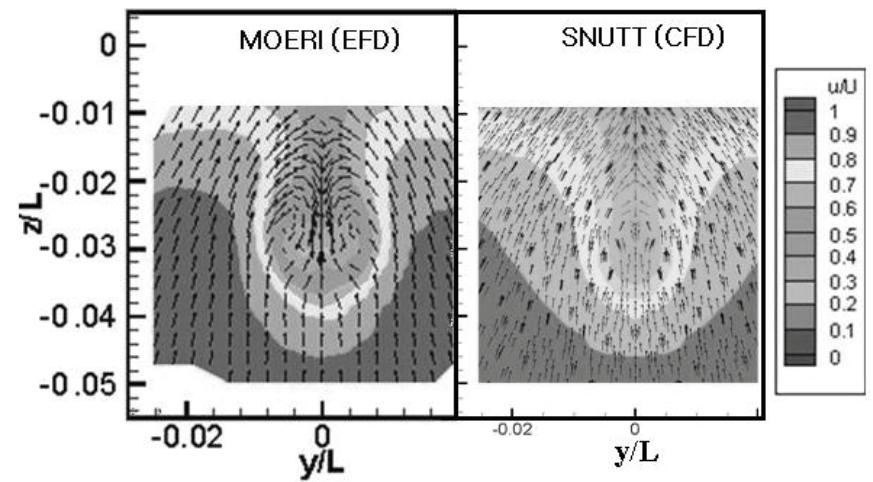

Fig. 10 Comparison of wake plane at $x / L_{p p}=0.4911$. 


\section{Propeller open water test}

The computational condition was set to the experimental one, i.e., the propeller revolution at 14rpswith varying flow speed to set the advance ratio, $J=V / n D$, to $0.1,0.3,0.5,0.7$, and 0.9 . The range of Reynolds number according to $J$ was from $1.45 \times 10^{5}$ to $1.30 \times 10^{6}$. The computed $K_{T}$ and $K_{Q}$ values were compared to the experimental data from MOERI, as shown in Tables 4 and 5. The comparison of $K_{T}$ is better than that of $K_{Q}$, and it is believed to be due to the difficulty in accurately predicting the drag on the propeller blade, which is largely responsible for torque. In the course of the computation, it was learned that the wall $y^{+}$value should be less than 100 , even with the wall function boundary condition on the blade surfaces.

Table 4 Thrust Coefficient $\left(K_{T}\right)$.

\begin{tabular}{|c|c|l|l|}
\hline$J$ & $R n$ & $\begin{array}{l}\text { MOERI } \\
\text { (Exp.) }\end{array}$ & \multicolumn{1}{|c|}{ Present } \\
\hline 0.1 & $1.45 \times 10^{5}$ & 0.4763 & $0.4642(-2.53 \%)$ \\
\hline 0.3 & $4.33 \times 10^{5}$ & 0.3814 & $0.3829(0.39 \%)$ \\
\hline 0.5 & $7.22 \times 10^{5}$ & 0.2763 & $0.2744(-0.68 \%)$ \\
\hline 0.7 & $1.01 \times 10^{6}$ & 0.1770 & $0.1786(0.92 \%)$ \\
\hline 0.9 & $1.30 \times 10^{6}$ & 0.0757 & $0.0721(-4.67 \%)$ \\
\hline
\end{tabular}

Table 5 Torque Coefficient $\left(K_{Q}\right)$.

\begin{tabular}{|c|c|c|c|}
\hline$J$ & $R n$ & $\begin{array}{l}\text { MOERI } \\
\text { (Exp.) }\end{array}$ & Present \\
\hline 0.1 & $1.45 \times 10^{5}$ & 0.6720 & $0.6595(2.08 \%)$ \\
\hline 0.3 & $4.33 \times 10^{5}$ & 0.5527 & $0.5732(5.05 \%)$ \\
\hline 0.5 & $7.22 \times 10^{5}$ & 0.4258 & $0.4583(3.62 \%)$ \\
\hline 0.7 & $1.01 \times 10^{6}$ & 0.2992 & $0.3316(7.12 \%)$ \\
\hline 0.9 & $1.30 \times 10^{6}$ & 0.1682 & $0.1929(6.30 \%)$ \\
\hline
\end{tabular}

Figs. 11 and 12 show the surface pressure coefficient distribution at $J=0.7$ and 0.9 , respectively. The general tendency of surface pressure distribution on propeller blade surfaces was well reproduced, and the regions of extremely high and low pressure were clearly identified.
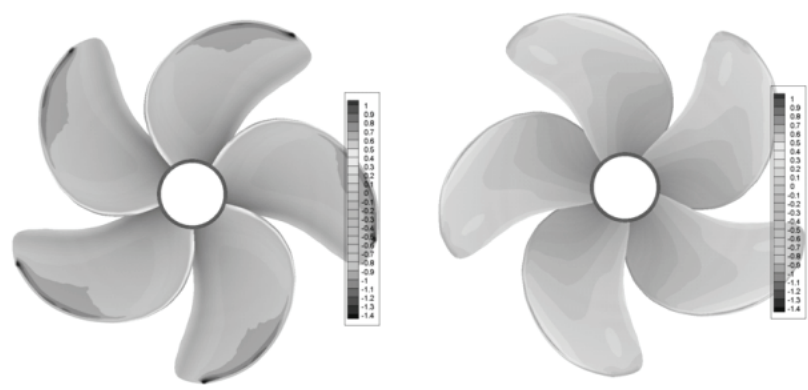

Fig. 11 Pressure distributionat $J=0.7$ (left: back side, right: face side).
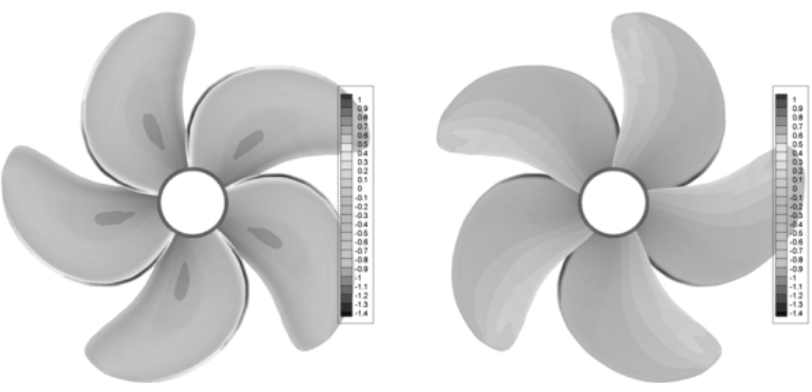

Fig. 12 Pressure distribution at $J=0.9$ (left: back side, right: face side).

\section{Self-propulsion test}

It was confirmed, from the above propeller open water test, that the use of the sliding mesh technique can produce reliable solutions for rotating propellers. The same approach was adopted for self-propulsion test with the rotating cylinder placed at the propeller location.

The computation was started with non-rotating propeller to first develop the steady flow field around the hull. Then the propeller was slowly rotated, and the revolution was gradually increased to the desired speed. The time step size was determined to make Courant number no smaller than 0.5. By rough estimate, the propeller revolution speeds of 9.0, 9.5, and $10.0 \mathrm{rps}$ were considered, and the self-propulsion point was found by linear curve fitting using those three revolution speeds. For the full scale extension, as for the towing tank tests, the towing force $\left(F_{D}\right)$ was considered to adjust the difference of the viscous force between the model and full scale ships.

The computational results for the three revolution speeds are listed in Table 6 , where the error value is defined as $\left(R_{T}-T-F_{D_{0}}\right) / 0.5 \rho U^{2} S_{M}$. Based on these results, the selfpropulsion point was searched using a linear interpolation, and the obtained revolution speed, $K_{T}$, and $K_{Q}$ are presented in Table 7.

The pressure distribution on the hull surface and wake distribution at $x / L_{p p}=0.4911$ are shown in Figs. 13 15, with comparison to the experimental data. The pressure recovery around the bilge was well predicted both quantitatively and qualitatively. Also the effects of rotating propeller in the hull wake conform to those observed in the experiment.

Table 6 Calculation results of three rps cases.

\begin{tabular}{|c|c|c|c|c|}
\hline$r p s$ & $J$ & $K_{T}$ & $K_{Q}$ & error \\
\hline 9.0 & 0.9761 & 0.1452 & 0.0281 & $5.88 \times 10^{-4}$ \\
\hline 9.5 & 0.9247 & 0.1721 & 0.0307 & $-9.92 \times 10^{-5}$ \\
\hline 10.0 & 0.8785 & 0.1903 & 0.0331 & $-3.95 \times 10^{-4}$ \\
\hline
\end{tabular}

Table 7 Result parameters of self propulsion test.

\begin{tabular}{|c|c|c|}
\hline$r p s$ & $K_{T}$ & $K_{Q}$ \\
\hline 9.592 & 0.1700 & 0.03110 \\
\hline
\end{tabular}


The pressure distribution on the hull surface and wake distribution at $x / L_{p p}=0.4911$ are shown in Figs. 13-15, with comparison to the experimental data. The pressure recovery around the bilge was well predicted both quantitatively and qualitatively. Also the effects of rotating propeller in the hull wake conform to those observed in the experiment.

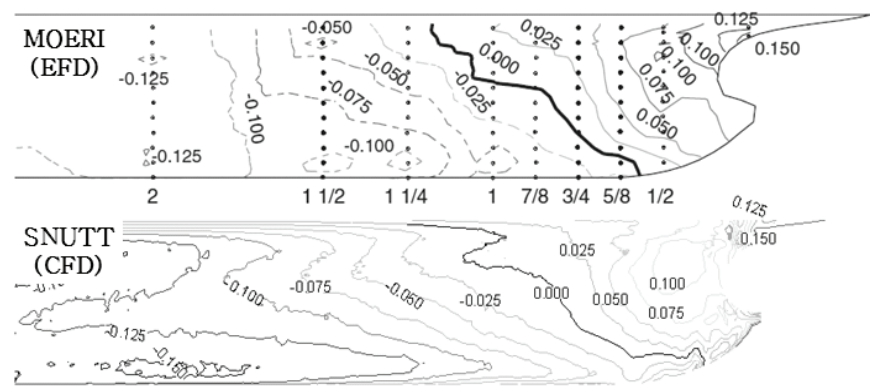

Fig. 13 Comparison of hull surface pressure distribution.

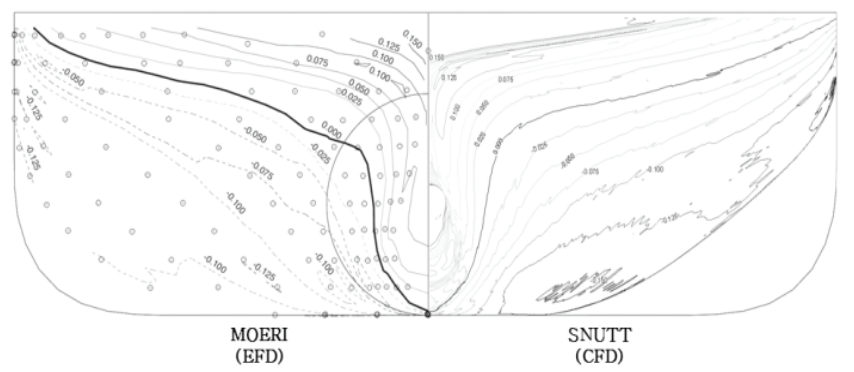

Fig. 14 Comparison of pressure distribution(port side).

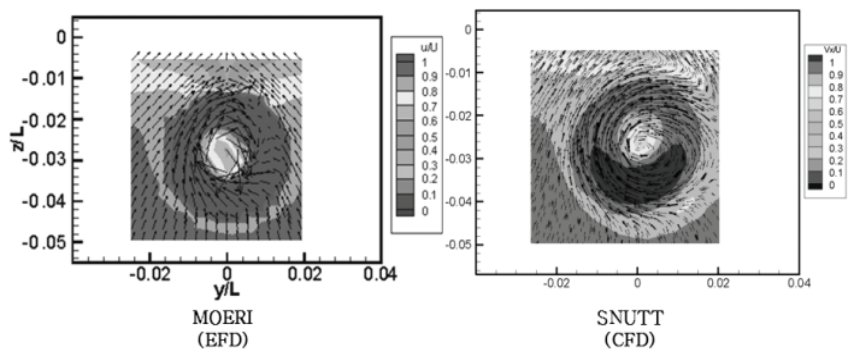

Fig. 15 Comparison of wake plane at $x / L_{p p}=0.4911$.

Lastly, the quasi-propulsive coefficient $\left(\eta_{D}\right)$ was derived using the results from resistance, propeller open water, and self-propulsion tests. Table 8 presents the self-propulsion parameters obtained from the experimental data from NMRI and other computational results reported at the CFD Workshop Tokyo 2005 (Tahara et al., 2005; Karl and Chao, 2005;Lübke, 2005; Kim et al., 2005)

Compared to the experimental data and other computational results, the present results show underprediction of $\eta_{\mathrm{D}}$. It is mainly attributed to the excessive numerical diffusion in the stern region caused by the tetrahedral cells there. However, it should be also noted that most of the other computational results use the body force momentum source terms to represent the propeller effects in self-propulsion test, which must have a significant influence on the thrust deduction factor prediction. It is recommended to refine the mesh generation procedure for the stern region for better prediction of wake and propeller flow there.

Table 8 Computed self-propulsion parameters.

\begin{tabular}{|c|c|c|c|c|c|c|c|}
\hline & $1-t$ & $1-w_{t}$ & $\eta_{0}$ & $\eta_{r}$ & $J$ & $n$ & $\eta_{D}$ \\
\hline $\begin{array}{c}\text { NMRI } \\
\text { (Exp.) }\end{array}$ & 0.835 & 0.792 & 0.682 & 1.011 & 0.728 & 9.50 & 0.740 \\
\hline MOERI & 0.846 & 0.779 & 0.671 & 1.023 & 0.729 & 9.38 & 0.746 \\
\hline HSVA & 0.865 & 0.789 & 0.667 & 0.981 & 0.725 & 9.56 & 0.717 \\
\hline SVA & 0.910 & 0.765 & 0.614 & 1.007 & 0.708 & 9.50 & 0.618 \\
\hline OPU & 0.852 & 0.789 & 0.631 & 1.074 & 0.718 & 9.53 & 0.732 \\
\hline Present & 0.833 & 0.773 & 0.613 & 1.004 & 0.708 & 9.59 & 0.665 \\
\hline
\end{tabular}

\section{CONCLUSIONS}

In the present study, flexible meshing techniques, i.e., hybrid meshing for complex geometry regions and sliding mesh for rotating propeller, were employed to perform the three primary tests for ship resistance and propulsion performance, i.e., resistance, propeller open water, selfpropulsion tests. Unstructured meshing for the bow and stern regions, together with structured meshing for the remaining region, was used and non-conformal interfaces were placed between the sub-domains with different cell types. The sliding mesh technique, where the propeller was placed in a rotating cylindrical volume with non-conformal interfaces between the rotational and stationary sub-domains, was used to represent the rotating propeller in open water and selfpropulsion tests.

The computational results were compared with experimental data and other computational results. The overall performance of the flexible meshing techniques was commendable, but with slight under-predicted wake and quasi-propulsive coefficients. Development of the refinement of mesh generation procedure for the stern region is recommended for future work.

\section{ACKNOWLEDGMENT}

This research was supported by National Research Foundation (Grant No. 20090093129) and World Class University project (R32-2009-000-10161-0) funded by the Ministry of Education, Science and Technology of Korea government.

\section{REFERENCES}

Chao, Y. and Karl, K., 2005. Numeric Propulsion Simulation for the KCS Container Ship. Proceedings of CFD workshop Tokyo 2005, Tokyo, Japan, pp.539-545. 
Jasak, H., 2009. OpenFOAM: Open Source CFD Research and Industry. International Journal of Naval Architecture and Ocean Engineering, 1(2), pp.89-94.

Kim, M.-C. Park, W.-G. Chun, H.-H. and Jung, U.-H., 2010. Comparative Study on the Performance of POD Type Waterjet by Experiment and Computation. International Journal of Naval Architecture and Ocean Engineering, 2(1), pp.1-13.

Kim, J. Park, I.R. and Van, S.H., 2005. RANS Computations for KRISO Container Ship and VLCC Tanker using the WAVIS code. Proceedings of CFD workshop Tokyo 2005, Tokyo, Japan, pp.598-603.

Lee, J.H. Park, B.J. Seol, D.M. Rhee, S.H. Jun, D.S. Chi, H.R. and Ryu, M.C., 2009. Hybrid Meshing Approach for Resistance Performance Prediction of a POD Propulsion Cruise Ship. Proc. of ASCHT09, 2nd Asian Sympo. on Computational Heat Transfer and Fluid Flow, Jeju, Korea.
Lübke, L., 2005. Numerical simulation of the Flow around the propelled KCS. Proceedings of CFD workshop Tokyo 2005, Tokyo, Japan, pp.587-592.

Seo, D.W. Lee, S.-H. Kim, H. and Oh, J.K., 2010.A Numerical Study for the Efficacy of Flow Injection on the Diminution of Rudder Cavitation. International Journal of Naval Architecture and Ocean Engineering, 2 (2), pp.104-111.

Tahara, Y. Wilson, R. and Carrica, P., 2005. Comparison of Free-Surface Capturing and Tracking Approaches in Application to Modern Container Ship and Prognosis for Extension to Self-Propulsion Simulator. Proceedings of CFD workshop Tokyo 2005, Tokyo, Japan, pp.604611.

Yang, J. Rhee, S.H. and Kim, H., 2009. Propulsive Performance of a Tanker Hull Form in Damaged Conditions. Ocean Engineering, 36( 2), pp.133-144. 